\title{
EXISTENCE ET ARBITRARIÉTÉ DES CONNEXIONS COMPATIBLES À UNE STRUCTURE RIEMANN GÉNÉRALISÉE DU TYPE PRESQUE $k$-HORSYMPLECTIQUE MÉTRIQUE
}

\author{
PAR Radu Miron et GHeorghe Atanasiu
}

Dans les notes [12], [13] nous avons abordé le problème d'Eisenhart pour les espaces Riemann généralisés du type presque 0 - et 1-horsymplectiques métriques, établissant l'existence et l'arbitrariété des solutions. Notre attention porte à présent sur le cas général de la structure Riemann généralisée du type presque $k$-horsymplectique métrique. On associe à une telle structure, $g=g+g$, deux distributions $V$ et $H$ sur $M$, supplémentaires, orthogonales, par l'intermédiaire desquelles on détermine un champ de tenseurs antisymétriques $\stackrel{g}{g}$ du type $(2,0)$ et une structure presque $k$-horsymplectique locale. La condition de permutabilité (2.1) des opérateurs Obata appartenant à la structure $g$ montre que la structure presque $k$-horsymplectique locale mentionnée est également une structure métrique. Deux exemples justifient l'existence de telles structures [4], [5]. Les propriétés mentionnées servent à établir l'existence (théorème 6) et l'arbitrariété des connexions linéaires compatibles à $g$ (théorèmes 8 et 10 ). On y constate l'apparition de deux cas importants : elliptique et hyperbolique, selon que l'application $\mu: M \rightarrow R$, de (2.2), qui est constante, est positive ou négative. Les théorèmes 7 et 9 donnent les formes canonniques des connexions linéaires compatibles à $g$, pour chaque cas à part. Finalement, observons encore que les théorèmes 8 et 10 offrent toutes les connexions linéaires compatibles aux structures Riemann généralisées du type presque $k$-horsymplectique métrique elliptique ou hyperbolique.

Les notations et la technique de la démonstration des propriétés énumérées sont celles que nous avons utilisées dans les notes précédemment indiquées [12], [13].

\section{§1. Structures Riemann généralisées d'indice $k$}

Soit $M$ une variété différentiable de dimension $2 n+k$. Un champ de tenseurs $g \in \tau_{2}^{0}(M)$ a la décomposition unique dans la partie symétrique $g$ et la partie antisymétrique $g$

Received October 8, 1982 


$$
g=\underline{g}+g \text {. }
$$

Le champ g détermine une structure Riemann généralisée si

$$
\text { dét }\|\underline{g}\| \neq 0 \text {. }
$$

DÉfINITION 1. On considérera que la structure Riemann généralisée est d'indice $k$ si

$$
\text { rang }\|\underset{V}{g}\|=\operatorname{dim} M-k=2 n \text {. }
$$

Il est évident que $g$ détermine une structure Riemann sur $M$ et $g$ est une 2 -forme sur $M$. On montrera que $g$ détermine d'une mamière unique un champ de tenseurs $\check{g} \in \tau_{0}^{2}(M)$.

Vraiment, pour tout champ de tenseurs $X \in \mathfrak{X}(M)$, l'application

$$
\varphi: X \longrightarrow i_{X} g
$$

définit un morphisme du fibré tangent $T(M)$ au fibré cotangent $T^{*}(M)$, qui est homomorphisme sur des fibres. Soit $V=\operatorname{ker} \varphi . V$ est une distribution sur $M$ de dimension locale $k$. On note par $H$ la distribution supplémentaire, orthogonale à $V$, l'orthogonalité étant établie par rapport à $g$.

Dans chaque point $x \in M$ on a la décomposition directe

$$
T_{x}(M)=V_{x} \oplus H_{x}
$$

et $V_{x} \perp H_{x}$.

On note avec $v$ et $h$ les projecteurs (supplémentaires) déterminés par les distributions $V$ et $H$.

Soit $\left(U, x^{i}\right)$ un système de coordonnées locales sur $M$ et $g_{\imath \jmath}, g_{\imath \jmath}, g_{\imath \jmath}$ les coordonnées locales des champs $g$, g et respectivement $\underset{V}{g}$. Si $\xi_{1}^{\imath}, \cdots, \xi_{k}^{i}$ est une base locale orthonormée de $V$, alors

$$
g_{\vee a} \xi_{a}^{\jmath}=0, \quad g_{-a}^{\imath \jmath} \xi_{b}^{i \xi^{\jmath}}=\delta_{a b} \quad(a, b=1, \cdots, k) .
$$

On considère les champs duels

$$
\stackrel{a}{\eta_{2}}=\underset{-a}{g_{\imath} \xi} \xi^{\jmath} .
$$

On a donc:

$$
\underset{\substack{a \\ \eta_{i} \\ \xi^{\jmath}}}{=} \delta_{b}^{a}
$$

et les champs $v, h$ définis sur la variété $M$, ont les composantes locales:

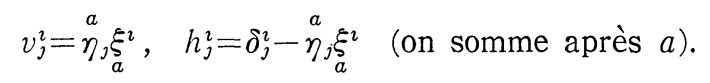

THÉORÈme 1. Il existe sur $M$ un seul champ de tenseurs, antisymétriques, du

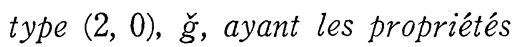




$$
i_{X} g \cdot \check{g}=h(X), \quad v \cdot \check{g}=0, \quad \forall X \in \mathscr{X}(M)
$$

Vraiment, le système d'équations en $g_{\vee}^{\imath \jmath}$ (les coordonnées de $\check{g}$ ):

$$
\begin{aligned}
& g_{i k} g_{\vee}^{i k}=h_{\imath}^{\jmath} \\
& a \vee \\
& \eta_{j} g \vee v=0,
\end{aligned}
$$

a la matrice (antisymétrique), non-singulière $\left\|\begin{array}{cc}g_{2 j}-\eta_{i} \\ a^{V} & \\ \eta_{j} & 0\end{array}\right\|$. Le champ $\check{g}$ ne dépend pas de la base locale $\underset{a}{\left(\xi^{i}\right)}$ de la distribution $V$.

OBSERVATION 1. Le triplet $(g, \underset{V}{\eta}, \underset{a}{\xi})$ définit une structure presque $k$-horsymplectique, locale.

On a, effectivement, du théorème précédent:

$$
\begin{aligned}
& g_{i k} g_{\vee}^{j k}+\underset{\eta_{i}}{a} \xi_{a}^{j}=\delta_{i}^{j} \quad \text { (on somme après } a \text { ) } \\
& \underset{b}{a} \xi_{b}^{\jmath}=\delta_{b}^{a} \\
& g_{\vee a} g_{\substack{2 j \\
\xi^{j}}}=0, \quad \eta_{j} g_{\vee}{ }^{2 \jmath}=0 \quad(a, b=1, \cdots, k) .
\end{aligned}
$$

Revenant à la structure $g$, on peut considérer les opérateurs Oproiu appartenant à $g$, [16].

$$
\begin{aligned}
& \phi_{i}^{r s}=\frac{1}{2}\left(\delta_{i}^{r} \delta_{j}^{s}+v_{i}^{r} v_{j}^{s}-g_{\vee} g_{V}^{s r}\right) \\
& \phi_{2}^{r s}=\frac{1}{2}\left(\delta_{i}^{r} \delta_{j}^{s}-v_{i}^{r} v_{j}^{s}+g_{\vee j} g_{V}^{s r}\right) \\
& \Theta_{i j}^{r s}=\frac{1}{2}\left(v_{i}^{r} h_{j}^{s}+h_{i}^{r} v_{j}^{s}\right)
\end{aligned}
$$

et les opérateurs Obata [15] de $g$ :

$$
\Lambda_{1}^{r s}=\frac{1}{2}\left(\delta_{i}^{r} \delta_{j}^{s}-g_{\underline{\imath} j} g^{r s}\right), \quad \Lambda_{2}^{r s}=\frac{1}{2}\left(\delta_{i}^{r} \delta_{j}^{s}+g_{\underline{\imath j}} g^{r s}\right) .
$$

On note par $\Lambda_{1} \cdot \phi_{1}$, ou $\Lambda_{1} \cdot \Theta$, le "produit" des opérateurs $\Lambda_{1}$ et $\phi_{1}$, ou $\Lambda_{1}$ et $\Theta$. On a $\left(\Lambda_{1} \cdot \phi\right)_{l j}^{r s}=\Lambda_{1 m}^{k s} \phi_{1 j}^{r m}$, et l'effet de l'application d'un de ces opérateurs, par exemple $\phi$, sur un champ $t \in \tau_{2}^{1}(M)$ sera noté $\operatorname{par}(\phi \cdot t)_{{ }_{\imath}}^{s}=\phi_{1}^{m}{ }_{r}^{s} t_{m}^{r}$.

Grâce à un calcul direct on démontre le

THÉORÈme 2. (i) Les opérateurs (1.9), (1.10) sont des champs de tenseurs sur $M$.

(ii) $\Lambda_{1}, \Lambda_{2}$ sont des projecteurs supplémentaires sur $\mathscr{F}(M)$-le module $\tau_{2}^{1}(M)$.

(iii) $\Lambda_{u} \cdot \Theta=\Theta \cdot \Lambda_{u}(u=1,2)$.

(iv) ${ }_{1}^{u}-\Theta$ et $\phi_{2}^{u}+\Theta$ sont des projecteurs supplémentaires sur $\tau_{2}^{1}(M)$. 
(v) Si $\Lambda_{1} \cdot \phi_{1}=\phi_{1} \cdot \Lambda_{1}$, alors les projecteurs $\Lambda_{1}$ et $\phi_{1}-\Theta$ (respectivement $\Lambda_{2}$ et $\phi_{2}+\Theta$ ) sont permutables et $\Lambda_{1} \cdot(\phi-\Theta), \Lambda_{2} \cdot(\phi+\Theta)$ sont des projecteurs supplémentaires sur $\tau_{2}^{1}(M)$

Aussi apparaît-il comme une conséquence le

THÉORÈme 3. Si les opérateurs $\Lambda_{1}, \phi_{1}$ sont permutables, alors le système d'équations tensorielles dans le champ de tenseurs $X \in \tau_{2}^{1}(M)$.

$$
\left\{\begin{array} { l } 
{ \Lambda \cdot X = 0 } \\
{ ( \phi - \Theta ) \cdot X = 0 }
\end{array} \left[\text { resp. }\left\{\begin{array}{l}
\Lambda \\
2 \\
(\phi+X=0 \\
\left.\phi_{2}+\Theta\right) \cdot X=0
\end{array}\right]\right.\right.
$$

a des solutions non-triviales et sa solution générale est

$$
X=\Lambda_{2} \cdot(\phi+\Theta) \cdot Y \quad\left[\text { resp. } X=\Lambda_{1} \cdot(\phi-\Theta) \cdot Y\right],
$$

où $Y \in \tau_{2}^{1}(M)$ est complètement arbitraire.

On appliquera ces résultats à la détermination de toutes les connexions linéaires compatibles à la structure $g$.

\section{$\S 2$. Structures Riemann généralisées du type presque $k$-horsymplectiques métriques}

DÉFINITION 2. La structure Riemann généralisée $g$ s'appelle du type presque $k$-horsymplectique métrique, si $g$ est d'indice $k$ et les opérateurs $\Lambda_{1}, \phi_{1}$ sont permutables :

$$
\Lambda_{1} \cdot \phi=\phi_{1} \cdot \Lambda_{1}
$$

THÉORÈme 4. La structure Riemann généralisée $g$, d'indice $k$, est presque k-horsymplectique métrique, si et seulement s'il existe une fonction non-nulle $\mu: M \rightarrow R$, de telle manière qu' on ait

$$
g_{\underline{\imath \underline{r}}} g_{\underline{\jmath s}} g_{\mathrm{V}}^{r s}=\mu g_{\underline{\imath}} \text {. }
$$

Démonstration. L'équation (2.1) est équivalente à

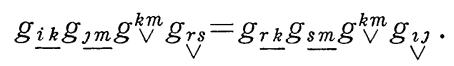

Si on note

$$
c_{\imath \jmath}=g_{\underline{\imath \underline{r}}} g_{\underline{\jmath} \underline{m}} g_{\vee}^{r m},
$$

la dernière égalité nous offre $c_{\imath j} g_{r s}=c_{r s} g_{\vee \jmath}$. Par contraction avec $g_{V}^{r s}$ et notant avec $\mu=(1 / 2 n) c_{r s} g_{V}^{r s}$, on obtient (2.2). Toujours de (2.2) on s'aperçoit que $\mu \neq 0$ sur $M$. La propriété réciproque est immédiate. 
EXEMPLE 1. L'ensemble d'objets géométriques $\left(F_{j}^{i}, \eta_{\imath}^{a}, \underset{a}{\xi_{2}}, g_{\underline{\imath} j}\right)(a=1, \cdots, k)$, où $F_{j}^{i}$ est un champ de tenseurs du type $(1,1)$ sur $M, \stackrel{a}{\eta_{\imath}}$ sont des $k$ champs de covecteurs sur $M, \underset{a}{\xi_{a}^{2}}$ sont des $k$ champs de vecteurs sur $M$ et $g_{\underline{\imath j}}=g_{\underline{j i}}$ est une structure Riemann sur $M$, présentant les propriétés:

$$
\begin{aligned}
& F_{r}^{i} F_{j}^{r}=-\delta_{j}^{2}+{\stackrel{\eta}{\eta}, \xi_{a}^{2}}^{2} \quad \text { (on somme après } a \text { ) } \\
& F_{j}^{i}{ }_{j}^{a}=0 \text {, } \\
& F_{j}^{i} \xi_{a}^{j}=0, \quad \stackrel{a}{\eta_{j} \xi_{b}^{j}=\delta_{b}^{a},} \underset{a}{a}, \\
& F_{i}^{r} F_{j}^{s} g_{\underline{r s}}=g_{\underline{\imath j}}-\stackrel{\eta}{\eta}_{i} \eta_{j} \text { (on somme après } a \text { ) }
\end{aligned}
$$

sera appelé une $\left(F_{j}^{\imath}, \stackrel{a}{\eta_{l}}, \underset{a}{\xi^{2}}, g_{\underline{i j}}\right)$-structure elliptique sur $M$.

$\mathrm{Au}$ cas de $k=2, \mathrm{~K}$. Yano et quelques collaborateurs [20] ont prouvé l'existence de telles structures sur certaines variétés $M$, de dimension $2 n+2$.

S'il existe une $\left(F_{\jmath}^{\imath}, \eta_{\imath}^{a}, \xi_{a}^{\imath}, g_{\underline{\imath} \underline{j}}\right)$-structure elliptique sur $M,(a=1, \cdots, k)$, alors prenant $g_{\vee \jmath}=F_{i}^{r} g_{\underline{\jmath}}$, le champ de tenseurs $g_{\imath \jmath}=g_{\underline{\imath j}}+g_{\vee \mathfrak{V}}$ détermine une structure presque $k$-horsymplectique métrique (elliptique) sur $M$.

EXEMPLE 2. L'ensemble d'objets géométriques $\left(P_{\jmath}^{\imath}, \underset{\eta_{\imath}}{a} \underset{a}{\xi^{2}}, g_{i j}\right)(a=1, \cdots, k)$ où $P_{j}^{\imath}$ est un champ de tenseurs du type $(1,1)$ sur $M, \stackrel{a}{\eta_{2}}$ sont des $k$ champs de convecteurs sur $M, \xi_{a}^{\xi^{2}}$ sont des $k$-champs de vecteurs sur $M$ et $g_{\underline{2} \underline{j}}=g_{\underline{j i}}$ une structure Riemann sur $M$, ayant les propriétés

$$
\begin{aligned}
& \left.P_{r}^{\imath} P_{j}^{r}=\delta_{j}^{2}-\eta_{j}^{a} \xi_{a}^{\imath} \quad \text { (on somme après } a\right) \\
& P_{j}^{a} \eta_{\imath}=0, \quad P_{j}^{i} \xi_{a}^{j}=0, \quad{ }^{a} \eta_{j} \xi_{b}^{j}=\delta_{b}^{a}, \\
& \left.P_{i}^{r} P_{j}^{s} g_{\underline{r s}}=-g_{\underline{i} j}+\eta_{i} \eta_{j} \quad \text { (on somme après } a\right)
\end{aligned}
$$

sera nommé une $\left(P_{\jmath}^{\imath}, \stackrel{a}{\eta}_{\imath}, \xi_{a}^{\jmath}, g_{\underline{\imath}}\right)$-structure hyperbolique sur $M$.

Une structure presque hermitienne hyperbolique, [11] induit sur une sousvariété de co-dimension 2 appartenant à $M$ une $\left(P_{\jmath}^{\imath}, \stackrel{a}{\eta_{\imath}}, \xi_{a}^{\jmath}, g_{\underline{2 j}}\right)$-structure hyperbolique $(a=1,2)$.

S'il existe sur $M$ une $\left(P_{\jmath}^{\imath}, \stackrel{a}{\eta_{\imath}}, \underset{a}{\xi_{a}^{2}}, g_{\underline{\imath} \underline{j}}\right)$, structure hyperbolique, $(a=1, \cdots, k)$, alors prenant $g_{\mathfrak{v}}=P_{i}^{r} g_{\underline{\jmath x}}$ on obtient $g_{\imath \jmath}=g_{\underline{\imath} j}+g_{\underline{\imath}}$ qui est une structure Riemann généralisée du type $k$-horsymplectique métrique (hyperbolique).

Ces deux exemples prouvent l'existence des structures Riemann généralisées du type $k$-horsymplectiques métriques $g$ (elliptiques et hyperboliques) et en même temps ils suggèrent la méthode moyennant laquelle on obtient les connexions compatibles à la structure $g$. 
A ce qu'on pourra remarquer, la fonction $\mu$ de (2.2) est constante. De ce fait on peut donner:

DÉfinition 3. La structure Riemann généralisée $g$ du type presque $k$-horsymplectique métrique s'appelle: elliptrque si $\mu=c^{2}(c>0, c \in R)$ et hyperbolique si $\mu=-c^{2}(c>0, c \in R)$.

$\mathrm{Au}$ cas elliptique l'équation (2.2) est équivalente à

$$
c g \stackrel{2 r}{g_{J r}}=-\frac{1}{c} g_{\vee}^{\imath r} g_{\underline{J r}}
$$

et on peut considérer le champ de tenseurs du type $(1,1)$ :

$$
F_{\jmath}^{\imath}=c g_{\stackrel{\imath r}{\imath}} g_{\mathrm{V}} \text {. }
$$

$\mathrm{Au}$ cas hyperbolique l'équation (2.2) est équivalente à

$$
c g \stackrel{2 r}{g_{\mathfrak{J}}}=\frac{1}{c} g_{\vee}^{2 r} g_{\underline{J r}}
$$

et il est possible de considérer le champ de tenseurs du type $(1,1)$ :

$$
P_{j}^{\imath}=c g_{\stackrel{\imath r}{\imath}} g_{\mathrm{V}}
$$

Proposition 1. Le champ $F_{j}^{\imath}$ de la structure g elliptrque et le champ $P_{j}^{\imath}$ de la structure $g$ hyperbolique, sont globalement définis sur $M$.

Observatıon 2. L'ensemble $\left(F_{\jmath}^{i}, \eta_{\jmath}^{a}, \xi_{a}^{\imath}, g_{\underline{\imath} j}\right)$ donné par (1.4), (1.5) et (2.3) détermine une $\left(F_{\jmath}^{\imath}, \stackrel{a}{\eta_{\imath}}, \xi_{a}^{\imath}, g_{\underline{\imath}}\right)$-structure elliptique locale.

Observation 3. L'ensemble $\left(P_{\jmath}^{\imath}, \stackrel{a}{\eta_{\jmath}}, \underset{a}{\xi^{2}}, g_{\underline{i j}}\right)$-donné par (1.4), (1.5) et (2.4) détermine une $\left(P_{\jmath}^{\imath}, \stackrel{a}{\eta_{\imath}}, \xi_{a}^{\imath}, g_{\imath \underline{j}}\right)$ structure hyperbolique locale.

\section{$\S 3$. Connexions linéaires compatibles à une structure $g$ du type presque $k$-horsymplectique métrique}

Le problème important est certes celuı de l'existence des connexions linéaires $\Gamma$ par rapport auxquelles la structure Riemann généralisée $g$ est parallèle.

Définition 4. Nous considérerons qu'une connexion linéaire $\Gamma$ est compatible à la structure Riemann généralisée $g$ si $\nabla_{X} g=0, \forall X \in \mathscr{X}(M)$.

On constate immédiatement que $\Gamma$ est compatible a $g$ si et seulement si :

$$
g_{\underline{\imath} j \mid k}=0, \quad g_{\mathfrak{V} \mid k}=0 \text {. }
$$

Un premier résultat est avancé par le

THÉORÈme 5. S'il exıste une connexıon linéarre $\Gamma$ compatible à une structure Riemann généraliséé $g$ du type presque k-horsymplectique métrıque sur $M$, alors. 
(i) les distributıons $V$ et $H$ sont parallèles par rapport à $\Gamma$ sur $M$;

(ii) les projecteurs $v, h$ et le champ ğ sont parallèles sur $M$;

(iii) la fonction $\mu$ de (2.2) est constante.

Démonstratıon (i). Selon (3.1) en appliquant la dérivation covariante $g_{\imath j} \xi_{a}^{2}=0$, on obtient $g_{\substack{2 j \\ \vee a}} \xi_{k}^{2}=0$. Donc $\underset{a}{\xi_{1 k}^{2}}$ appartiennent à la distribution $V$. Il s'ensuit que $V$ est parallèle par rapport à la connexion $\Gamma$ sur $M$. Si on applique de nouveau (3.1) il s'ensuit que $H$ est parallèle sur $M$.

(ii). Selon (i) il résulte $v$ et $h$ parallèles. De $g_{\imath} g_{V}^{3 r}=h_{\imath}^{3}$ en appliquant l'opérateur de dérivation covariante on parvient à

$$
g_{\vee r} g \vee{ }^{3 r}=0 \text {. }
$$

Si on réalise la contraction avec $g_{\vee}^{2 p}$, on a $g_{\vee \mid k}^{p p}-v_{s}^{p} g_{\vee}^{J s}{ }_{k}=0$, relation que nous pouvons écrire $\left.g_{\vee}^{\jmath p}\right|_{k}-\left(v_{s}^{p} g_{\vee}^{\jmath s}\right)_{\mid k}=0$. Par d'autres mots $g_{\vee{ }_{1 k}}^{\jmath 0}=0$.

(iii) $\mathrm{Si}$ on dérive d'une manière covariante (2.2), selon (3.1) et (ii) on a $\mu_{k} g_{V j}=0$, qui implique $\mu_{k}=0\left(\mu_{k}=\partial \mu / \partial x^{k}\right)$. Donc $\mu=$ constant.

Par conséquent on a à considérer les deux cas $\mu=c^{2}$ (elliptique) et $\mu=-c^{2}$ (hy perbolique).

THÉoRÈme 6. Sur la varlété différentiable $M$ douée d'une structure Riemann généralisée $g$ du type k-horsymplectıque métrique, il existe une connexion linéaire $\Gamma$ compatible à $g$, si et seulement si la structure $g$ est elliptique ou hyperbolique.

Démonstratıon. Si $\Gamma$ existe, conformément au théorème précédent la structure $g$ est elliptique ou hyperbolique.

Récıproquement: a) Supposons que g est elliptique. Soit $\stackrel{\Gamma}{\Gamma}$ une connexion linéaire sur $M$, fixée et $i$ la dérivation covariante par rapport à $\stackrel{\circ}{\Gamma}$. La connexion linéaire suivante sur $M$ est compatible à $g$ :

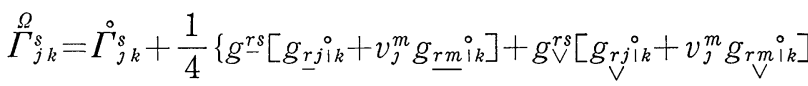

$$
\begin{aligned}
& +F_{\jmath}^{r}\left[F_{r i k}^{s \circ}+v_{m}^{s} F_{r \mid k}^{m \circ}\right]+v_{j}^{r} v_{r i k}^{s \circ}-v_{r}^{s} v_{\jmath \mid k}^{r \circ}+2 \Lambda_{1}^{r s}\left[3 v_{q}^{m} v_{r \mid k}^{q \circ}-v_{r \mid k}^{m \circ}\right],
\end{aligned}
$$

avec $F_{\jmath}^{\imath}$ donné par (2.3).

Assurément, par calcul direct on prouve que $\stackrel{\Omega}{\Gamma}$ satisfait à (3.1).

b) Si $g$ est hyperbolique, la connexion linéaire suivante est compatible à $g$ :

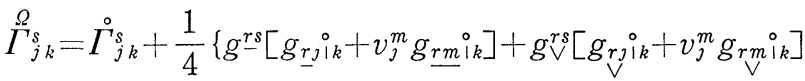

$$
\begin{aligned}
& -P_{j}^{r}\left[P_{r \mid k}^{s \circ}+v_{m}^{s} P_{r \mid k}^{m \circ}\right]+v_{j}^{r} v_{r \mid k}^{s \circ}-v_{r}^{s} v_{j \nmid k}^{r \circ}+2 \Lambda_{1}^{r s}\left[3 v_{q}^{m} v_{r i k}^{q \circ}-v_{r \mid k}^{m \circ}\right],
\end{aligned}
$$

où $P_{j}^{2}$ est donné par (2.4).

Evidemment, par calcul direct on prouve que $\stackrel{\Omega}{\Gamma}$ satisfait à (3.1). q.e.d. 
La connexion $\stackrel{\Omega}{\Gamma}$ donnée par (3.2), ou (3.3), sera appelée connexion compatible à $g$ associée à la connexion $\stackrel{\circ}{\Gamma}$.

\section{§4. Généralité de la solution du problème d'Eisenhart}

Ce qui intéresse c'est l'arbitrariété de la solution du problème d'Eisenhart.

DÉfinition 5. La connexion (3.2) respectivement (3.3) compatible à $g$ associée à la connexion $\stackrel{\circ}{\Gamma}_{j k}^{i}=\left\{\begin{array}{l}i \\ j k\end{array}\right\}$ déterminée par $g$ s'appelle la connexıon canonıque de la structure $g$.

On note cette connexion par $\stackrel{c}{\Gamma}$.

1) La structure Riemann généralisée $g$ du type presque $k$-horsymplectique métrique elliptique.

THÉORÈME 7. La connexion canonıque $\stackrel{c}{\Gamma}$ de la structure Rlemann généralisée $g$ du type presque k-horsymplectıque métrıque ellıptıque présente une des formes survantes équivalentes deux par deux:

(i ) $\Gamma_{j k}^{c}=\left\{\begin{array}{l}s \\ \jmath k\end{array}\right\}+\frac{1}{4}\left\{g_{\vee}^{r s} g_{V^{j} i_{k}}^{o}+F_{j}^{r} F_{r \mid k}^{s \circ}+2 \Lambda_{1}^{r s}\left(3 v_{q}^{m} v_{r \mid k}^{q \circ}-v_{r \mid k}^{m \circ}\right)\right\}$,

(ii) $\Gamma_{j k}^{c}=\left\{\begin{array}{l}s \\ \jmath k\end{array}\right\}+\frac{1}{2}\left\{g_{\vee}^{r s} g_{\underset{\gamma}{r j k}}^{o}+3 v_{r}^{s} v_{j \mid k}^{r o}-v_{j \mid k}^{s o}\right\}$,

(iii) ${\stackrel{c}{s}{ }_{j k}}^{\prime}=\left\{\begin{array}{l}s \\ \jmath k\end{array}\right\}+\frac{1}{2}\left\{F_{\jmath}^{r} F_{r \mid k}^{s \circ}+v_{\jmath \mid k}^{s \circ}-3 v_{j}^{r} v_{r \mid k}^{s o}\right\}$,

avec $F_{\jmath}^{2}$ donné par (2.3).

En appliquant le théorème 3 on prouve le:

THÉORÈmE 8. Toutes les connexions linéarres compatibles à une structure Rıemann généralisée $g$ du type presque k-horsymplectıque métrıque elliptıque sont données par la formule

$$
\Gamma_{j k}^{s}={\stackrel{c}{\Gamma_{j k}^{s}}}^{s}+\left[\Lambda_{1} \cdot(\phi-\Theta)\right]_{j m}^{r s} Y_{r k}^{m}
$$

où $\stackrel{c}{\Gamma}$ est la connexion canonique de la structure $g$, et $Y_{j k}^{\imath}$ est un champ de tenseurs du type (1,2), arbitraire.

2) La structure Riemann généralisée $g$ du type presque $k$-horsymplectique métrique hyperbolique.

THÉorème 9. La connexion canonique $\stackrel{c}{\Gamma}$ de la structure Riemann généralisée $g$ du type presque k-horsymplectique métrique hyperbolique possède une des formes suivantes équivalentes deux par deux. 


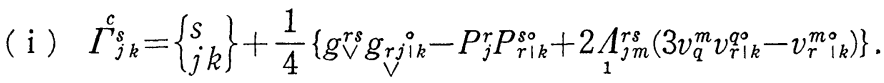

(ii) $\Gamma_{j_{k}}^{c}=\left\{\begin{array}{l}s \\ j k\end{array}\right\}+\frac{1}{2}\left\{g_{\vee}^{r s} g_{\left.\underset{r j \mid k}{o}+3 v_{r}^{s} v_{j k}^{r o}-v_{j \mid k}^{s o}\right\}}\right\}$

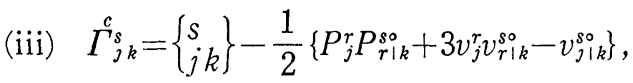

avec $P_{\jmath}^{\imath}$ donné par (2.4).

Faisant appel, de nouveau, au théorème 3 , on déduit le

THÉORÈmE 10. Toutes les connexions linéaires compatibles à une structure Rıemann généralisée $g$ du type presque k-horsymplectique métrique hyperbolique sont données par la formule (4.1) où $\stackrel{c}{\Gamma}$ est la connexion canonique du théorème 9 , et $Y_{j k}^{\imath}$ est un champ de tenseurs du type $(1,2)$ arbitrairement sur $M$.

\section{REFERENCES}

[1] C. DE Barros, Variétés horsymplectiques, C.R. Acad. Sc. Paris, 259 (1964), 12911294 .

[2] Blair, D. E. Ludden G.D. And K. Yano, Induced structures on submanifolds, Kōdai Math. Sem. Rep., 22 (1970), 188-198.

[3] L.P. Eisenhart, General Riemann spaces I, II, Proc. Nat. Sc. U.S. A., 37 (1951), $5 ; 38$ (1952), 6.

[4] S.I. GoldDBerg AND K. Yano, On normal globally framed $f$-manifolds, Tôhoku Math. J., 22 (1970), 362-370.

[5] S.I. GoldBerg AND K. YANO, Globally framed $f$-manifolds, I Illinois J. of. Math., 15 (1971), 456-474.

[6] S. IANUŞ, C. UDRIŞTE, Asupra spaţiului fibrat tangent al unei varietăţi diferențiabile, Stud. şi Cercet. Matem., 22, 4 (1970), 599-611.

[7] A. Jakubowicz and J. KlekowsKa, The necessary and sufficient condition for the existence of the unique connection of the two-dimensional generalized Riemann space, Tensor N.S., 20 (1969), 72-74.

[8] A. KAWAGUCHI, Beziehung zwischen einer metrischen linearen Ubertragung und einer nicht-metrischen in einem allgemeinen metrischen Raume, Akad. Wetensch.

[9] P. LibermanN, Sur les structures presque complexes et autres structures infinitésimales régulieres. Bull. Soc, Math., France, 83 (1953), 168-224.

[10] A. Lichnerowicz, Réductivité des algèbres d'automorphisme des structures symplectiques, cosymplectiques et des structures de contact, Rend. Math. Roma, 22 (1963), 197-244.

[11] R. Miron and Gh. Atanasiu, Hyperbolical almost Hermitian structures, Lucr. Col. Naț, Geom.-Top., Bușteni-Prahova, 27-3. iunie 1981.

[12] R. Miron and Gh, Atanasiu, Existence et arbitraire de connnexions compatibles aux structures Riemann généralisées du type hermitien (sous presse dans "Tenseur", Japon, en honneur à M. Kawaguchi).

[13] R. Minon And Gh. Atanasiu, Existence et aribtrarieté de connexions compatibles aux structures Riemann généralisées du type presque 1-horsymplectiques métri- 
ques (sous presse Supl. An. Univ. "Al. I. Cuza," tom. XXVIII, 1981).

[14] H. Nakagawa, On framed $f$-manifolds, Kōdai Math. Sem. Rep., 18 (1966), 293306.

[15] M. OBATA, Affine connections on manifolds with almost complex, quaternion or Hermitian structure, Jap. J. Math., 26 (1957), 43-77.

[16] V. Oproiu, Almost horsymplectic and conformal almost horsymplectic connexions, Revue Roum. Math. pures et appl., XIV, 10 (1969), 1585-1607.

[17] Oтsuki, T., Theory of affine connections of the space of tangent directions of a differential manifold I, II, III, Math., J. Okayama Univ., 7 (1957), 1-74, 95-122.

[18] C. UdRIŞTE, Diagonal lifts from a manifold to its tangent bundle, Rendiconti di Matematica (4), 9, VI (1976), 539-550.

[19] Gh. VRÂNCEANU, Opera matematică, 1-4, Editura Academiei, Bucureşti, 1969-1976.

[20] Kentaro Yano and Musafumi Okumura, On $(f, g, u, v, \lambda)$-structures, Kōdai, Math. Sem. Rep., 22 (1970), 401-423.

Universite "Al. I. CuzA"

Fac. DE Mathématiques 6600 IASSY, ROUMANiE
UNIVERSitE DE BRAŞOV

FAC. DE MAThÉmatiques

2200 Braşov, Roumanie 